\title{
Predictors of Disease Severity in Adult Covid-19 Patients Admitted in Mayo Hospital, Lahore, Pakistan
}

\author{
Tazeen Nazar, Bilal Aziz, Bilquis Shabbir, Furqan Saeed, Kashif Nawaz and Muhammad Nabeel \\ Department of East Medical Ward, King Edward Medical University, Mayo Hospital, Lahore, Pakistan
}

\begin{abstract}
Objective: To assess disease severity, based on clinical presentation and laboratory investigations.

Study Design: Observational study.

Place and Duration of Study: COVID-19 Isolation Unit of Mayo Hospital, Lahore from 15th March to 31st May, 2020.

Methodology: Four hundred and forty-five COVID-19 RT-PCR positive patients of either gender in age group of 18-80 years, admitted in isolation wards, high dependency units (HDUs) and intensive care units (ICUs) of the Hospital, were selected for the study via simple random sampling. Clinical presentations and laboratory investigations were recorded for all patients. Kruskal-Wallis test, Pearson Chi-square test, boxplots and ROC curve were used to analyse the data. A p-value $\leq 0.05$ was considered statistically significant.

Results: Out of the 445 cases, a male predominance 286 (64.3\%) was observed with majority of patients 324 (72.8\%) having mild disease, 73 (16.4\%) moderate, 25 (5.6\%) severe and 23 (5.2\%) having critical disease. D-dimer was considered to be the best discriminatory marker to assess disease severity with an overall accuracy of $92.1 \%$.

Conclusion: Fever, sore throat, shortness of breath, body aches, abdominal pain, anosmia and aguesia were the predominant symptoms in majority of patients belonging to different categories based on disease severity. Inflammatory markers like D-dimers and ferritin levels determined the overall disease severity with a high accuracy.
\end{abstract}

Key Words: Disease severity, Clinical presentation, Laboratory investigations, RT-PCR.

How to cite this article: Nazar T, Aziz B, Shabbir B, Saeed F, Nawaz K, Nabeel M. Predictors of Disease Severity in Adult Covid-19 Patients Admitted in Mayo Hospital, Lahore, Pakistan. J Coll Physicians Surg Pak 2021; 31(06):638-643.

\section{INTRODUCTION}

COVID-19 has taken the world by a storm. It has wrecked havoc with the lives of millions of people worldwide. As of $12^{\text {th }}$ April, 2021, 136 million people have been affected with 2.94 million deaths attributed to this deadly virus. ${ }^{1}$

The disease has been relapsing and recurring in waves, owing to its varied presentation from the involvement of respiratory tract to affecting cardiovascular, neurological, gastrointestinal, hepatic, musculoskeletal and almost every organ system of the body. ${ }^{2}$ COVID-19 affects people of all age groups. In China, the median age of patients was 30-79years with a male preponderance..$^{3-7}$ The virus mainly affected individuals having comorbidities like diabetes, hypertension, cardiovascular and cerebrovascular disorders. ${ }^{8-11}$ Following a heavy influx of COVID-19 patients with multiple and varied presentations, it was decided to carry out a study that would highlight the various abnormalities observed in the clinical, laboratory and radiological findings that predict the course of the disease in the admitted patients.

Correspondence to: Dr. Tazeen Nazar, Department of East Medical Ward, King Edward Medical University Mayo Hospital, Lahore, Pakistan

E-mail: tazeennazar@gmail.com

Received: October 05, 2020; Revised: April 15, 2021;

Accepted: April 28, 2021

DOI: https://doi.org/10.29271/jcpsp.2021.06.638
The idea was to generate data from the Pakistani population. At the time when this study was started, only limited international data was available. The study also intended to help the healthcare providers identify risk factors enabling them to devise a strategy that will minimise disease progression and formulate treatmentguidelines.

The objective of this study was to assess disease severity, based on clinical presentation and laboratory investigations.

\section{METHODOLOGY}

This observational study included 445 cases of COVID-19, admitted in Mayo Hospital, Lahore, from $15^{\text {th }}$ March to 31st May, 2020. Diagnosis was made based on PCR positive result and only those patients were enrolled in the study, and the date of PCR positivity was taken as first day of illness. The patients were categorised as mild, moderate, severe and critical, based on their initial presentation. The patient's disease was classified as mild, moderate, severe and critical, according to the guidelines issued by the Ministry of National Health Services, Regulations and Coordination, Government of Pakistan. ${ }^{12}$

Mild cases were those who had symptoms of COVID-19, like cough, shortness of breath or difficulty in breathing, fever, chills, repeated shaking with chills, muscle pain, headache, sore throat, loss of taste or smell, but without any hemodynamic compromise, need for oxygen or chest X-ray findings, oxygen 
saturation $\geq 94 \%$. Moderate disease patients were those who had hypoxia (Oxygen saturation $<94 \%$ but $>90 \%$ ) or chest Xray with infiltrates involving $<50 \%$ of the lung fields. Severe disease patients were those who had clinical signs of pneumonia (fever/cough) plus either respiratory rate $>30$, severe respiratory distress, $\mathrm{SpO}_{2} \leq 90 \%$ on room air, chest X-ray involving $>50 \%$ of lung fields. Critical category had either ARDS, multiorgan dysfunction or septic shock.

Informed written consent to be enrolled for the study purpose and data collection was taken from all the patients having mild and moderate disease and from the attendants in cases of severe and critical disease. All vitals, laboratory and radiological data throughout the course of illness, were noted from the medical records and the most deranged (highest or lowest) values of all the variables were taken to predict the outcome.

The data was analysed using IBMSPSS Statistics 20.0. The quantitative data was expressed as mean \pm S.D and median (IQR: Q1-Q3) and comparison was made among groups by using Kruskal-Wallis test as the data was skewed. Kolmogorov-Smirnov and Shapiro-Wilk's tests were used to test the normality of data. Boxplots were used to present duration of stay in the hospital as per status, and inflammatory markers as per severity of disease. ROC curve was used to determine cut-offs of d-dimers for discrimination of severity. Pearson Chi-square test was applied to see association of severity of disease with gender, history, symptoms and presence of chronic diseases, where expected counts were more than 5 in all cells. And likelihood ratio test was applied where assumption of expected frequencies was violated. P-values $\leq 0.05$ was considered significant.

\section{RESULTS}

Out of a total of 445 COVID-19 cases, average age was calculated to be $54.6 \pm 10.4$ years and $286(64.3 \%)$ of them were males. They were categorised into four groups as per disease category. A large majority, i.e. 324 (72.8\%) were labelled to have mild disease, $73(16.4 \%)$ moderate, $25(5.6 \%)$ severe, and $23(5.2 \%)$ of them were critical. The average age for male and female patients was not different overall being $55.4 \pm 10.3$ years for males and $53.3 \pm 10.4$ years for females. All groups predominantly had more males except moderate category that had $53.4 \%$ females. Also travel and contact history had significant association, with mild category having maximum cases with positive travel and contact histories. $(p<0.001$ and 0.003 , respectively).

The signs and symptoms also had significant association with severity except for cough, flu-like illness, diarrhea and vomiting with $p$-values $0.644,0.511,0.876$ and 0.550 , respectively. Sore throat, body aches, anosmia and aguesia had higher percent of positive cases in the mild category group (all values $p<0.001$ ). Fever, shortness of breath and abdominal pain were more common in patients falling in the severe and critical groups (all values $p<0.001$ ). All chronic diseases showed a significantasso- ciation with severity of disease except malignancy with $p$-value 0.064. Respiratory illness was more common among severe and critical cases, while diabetes was more common among moderate and severe cases. Hypertension was most prevalent among severe cases followed by moderate and critical. Ischemic heart disease was most prevalent among critical, while cerebro vascular disease (CVD), chronic kidney disease (CKD) and chronic liver disease (CLD) were most prevalent among severe cases. The history of smoking was least prevalent among mild and most among severe cases (Table I).

It was noticed that all the markers measured had a significant difference among four groups by severity of disease. Among vitals, the systolic and diastolic blood pressures were significantly low in critical cases, while relatively higher in severe cases. Respiratory rate and pulse were significantly higher in severe and critical cases. Hemoglobin, white blood cell counts, platelet counts were all significantly higher in critical cases. Basophils and eosinophils were significantly lower in mild and lymphocytes were lower in the severe category. In the liver profile, ALT and AST levels were significantly higher for critical, while alkaline phosphatase, albumin and bilirubin were high in mild cases but were all in the normal range so had no significant association with disease severity. Urea, BUN and creatinine were all significantly higher in severe disease group. All inflammatory markers increased significantly with severity of disease. Magnesium, potassium, calcium and phosphate were highest for severe, sodium was highest for mild. Prothrombin time, APTT and INR values differed among the various categories of disease but remained within the usual range (Table II).

It was noted that the duration of hospital stay was prolonged for cases with mild or moderate disease with a median stay of 20 and 24 days, respectively; while those with severe disease had a median stay of 7 days and critical only 3 days. The reason for prolonged stay in mild and moderate cases was the time taken for complete recovery and two consecutive COVID PCR negative reports. In severe and critical cases, the patients died during the 3-7 days period following admission.

Correlation of the inflammatory markers with severity of disease showed that LDH had a slow increase with increase in severity, whereas CPK was different but not very discriminatory. CKMB was equally raised among moderate and severe cases and low in mild but high in critical cases. CRP was also raised with severity of disease. D-dimers could be considered the best discriminatory marker (among 6 tested) for differentiating the severity of disease with serum ferritin level as the second best (Tablell).

When the cut-offs were derived by using ROC curve for $\mathrm{d}$-dimer levels, it was noticed that $98.8 \%$ of mild cases had levels $\leq 450$, $75.3 \%$ of moderate cases had d-dimer levels in the range of 451 - 990, and $87.0 \%$ of critical cases had d-dimer levels above 2900 . The overall accuracy of the marker to determine severity was $92.1 \%$ (Table III). Figure 1 shows comparison of ROC curves for serum ferritin and d-dimers. 
Table I: Distribution of cases for chronic diseases in relation to severity of disease.

\begin{tabular}{|c|c|c|c|c|c|c|c|c|c|c|}
\hline & & \multicolumn{8}{|c|}{ Group } & \multirow{3}{*}{ p-value } \\
\hline & & \multicolumn{2}{|c|}{ Mild } & \multicolumn{2}{|c|}{ Moderate } & \multicolumn{2}{|c|}{ Severe } & \multicolumn{2}{|c|}{ Critical } & \\
\hline & & $\mathbf{n}$ & $\%$ & $n$ & $\%$ & $\mathbf{N}$ & $\%$ & $\mathbf{n}$ & $\%$ & \\
\hline \multirow{2}{*}{ Any respiratory illness } & Yes & 11 & 3.4 & 9 & 12.3 & 5 & 20.0 & 8 & 34.8 & \multirow{2}{*}{$<0.001$} \\
\hline & No & 313 & 96.6 & 64 & 87.7 & 20 & 80.0 & 15 & 65.2 & \\
\hline \multirow{2}{*}{ Diabetes } & Yes & 32 & 9.9 & 23 & 31.5 & 8 & 32.0 & 3 & 13.0 & \multirow{2}{*}{$<0.001$} \\
\hline & No & 292 & 90.1 & 50 & 68.5 & 17 & 68.0 & 20 & 87.0 & \\
\hline \multirow{2}{*}{ HTN } & Yes & 37 & 11.4 & 31 & 42.5 & 14 & 56.0 & 9 & 39.1 & \multirow{2}{*}{$<0.001$} \\
\hline & No & 287 & 88.6 & 42 & 57.5 & 11 & 44.0 & 14 & 60.9 & \\
\hline \multirow{2}{*}{ IHD } & Yes & 7 & 2.2 & 7 & 9.6 & 2 & 8.0 & 5 & 21.7 & \multirow{2}{*}{0.001} \\
\hline & No & 317 & 97.8 & 66 & 90.4 & 23 & 92.0 & 18 & 78.3 & \\
\hline \multirow{2}{*}{ CVD } & Yes & 2 & 0.6 & 1 & 1.4 & 3 & 12.0 & 2 & 8.7 & \multirow{2}{*}{0.004} \\
\hline & No & 322 & 99.4 & 72 & 98.6 & 22 & 88.0 & 21 & 91.3 & \\
\hline \multirow{2}{*}{ CKD } & Yes & 8 & 2.5 & 12 & 16.4 & 11 & 44.0 & 4 & 17.4 & \multirow{2}{*}{$<0.001$} \\
\hline & No & 316 & 97.5 & 61 & 83.6 & 14 & 56.0 & 19 & 82.6 & \\
\hline \multirow{2}{*}{ CLD } & Yes & 11 & 3.4 & 1 & 1.4 & 5 & 20.0 & 3 & 13.0 & \multirow{2}{*}{0.003} \\
\hline & No & 313 & 96.6 & 72 & 98.6 & 20 & 80.0 & 20 & 87.0 & \\
\hline \multirow{2}{*}{ Malignancy } & Yes & 0 & 0.0 & 2 & 2.7 & 0 & 0.0 & 0 & 0.0 & \multirow{2}{*}{0.064} \\
\hline & No & 324 & 100.0 & 71 & 97.3 & 25 & 100.0 & 23 & 100.0 & \\
\hline \multirow{2}{*}{ Smoking } & Yes & 59 & 18.2 & 32 & 43.8 & 12 & 48.0 & 9 & 39.1 & \multirow{2}{*}{$<0.001$} \\
\hline & No & 265 & 81.8 & 41 & 56.2 & 13 & 52.0 & 14 & 60.9 & \\
\hline
\end{tabular}

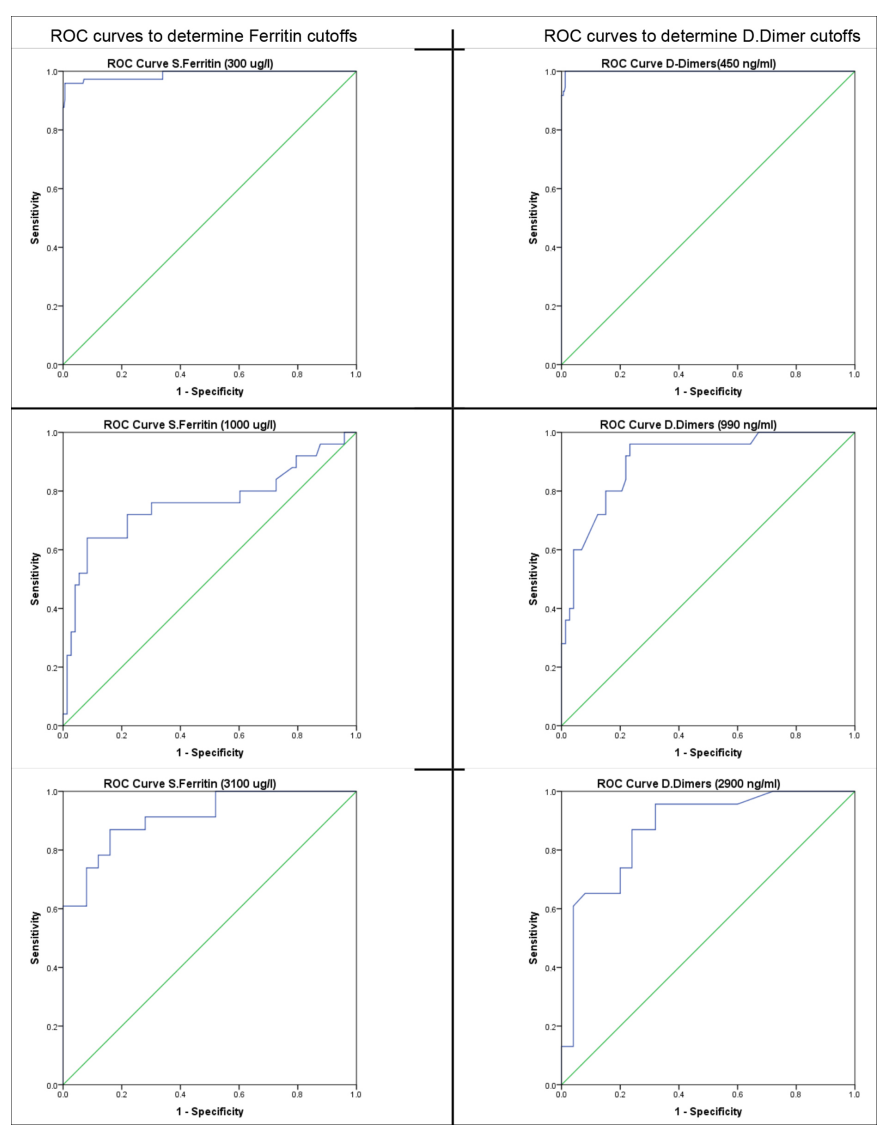

Figure 1: ROC curves for serum ferritin and d-dimers.

\section{DISCUSSION}

COVID-19 has had diverse presentations ever since the first case was admitted in the Hospital. Fever was the most common initial symptom presenting in the majority of patients, ${ }^{13-15}$ alongwith flu-like illness, cough and shortness of breath. With the passage of time, patients presented with headache, body aches, abdominal pain, diarrhea, anosmia and ageusia. These symptoms had a significant association with the severity of disease with sore throat, body aches, anosmia and ageusia being more common in the mild category, while patients with fever, shortness of breath and abdominal pain had a more severe disease. Smokers and patients with an underlying comorbid condition like diabetes, hypertension, ischemic heart disease, respiratory disease, CVD, CKD or CLD developed a more severe disease and; hence, the highest mortality. ${ }^{16}$ Apart from smoking, comorbidities were present in the majority of patients, the most common being hypertension followed by diabetes. ${ }^{17,18}$

Patients in the critical category also showed a high WBC count with a predominance of PMNs $(p<0.001)$ suggesting sepsis. Apart from neutrophilia, these patients also had leukopenia $(p<0.001)$ that contributed to a high mortality in this group. These results somewhat correlated with a study conducted by Yang Zhao et al. that suggested neutrophilia, leukopenia in addition to low $\mathrm{C} 3$ and $\mathrm{CD}^{+} \mathrm{T}$ cells to be the immunity-related risk factors that could predict a high mortality in COVID-19 patients. ${ }^{19}$ Further studies are needed to explain the role of these features and markers for a better understanding of the disease.

The biochemical profile of the patients, including the LFTs, and RFTs also had a role in assessing disease severity as these markers were found to be slightly higher in the critical and severe category compared to mild and moderate category of patients. This finding may partly be attributed to the underlying comorbid diseases like CLD, hypertension, diabetes and CKD or could possibly be due to the direct effect of the virus on these organ systems. 
Table II: Characteristics of cases as per severity of disease.

\begin{tabular}{|c|c|c|c|c|c|c|c|c|c|c|c|c|c|c|}
\hline & & \multicolumn{12}{|c|}{ Group } & \multirow{3}{*}{ p-value } \\
\hline & & \multicolumn{3}{|c|}{ Mild } & \multicolumn{3}{|c|}{ Moderate } & \multicolumn{3}{|c|}{ Severe } & \multicolumn{3}{|c|}{ Critical } & \\
\hline & & Median & Q1 & Q3 & Median & Q1 & Q3 & Median & Q1 & Q3 & Median & Q1 & Q3 & \\
\hline & Age & 55.0 & 48.0 & 62.0 & 54.0 & 49.0 & 63.0 & 60.0 & 57.0 & 65.0 & 58.0 & 47.0 & 65.0 & 0.006 \\
\hline & Days after diagnosis & 20.0 & 19.0 & 21.0 & 23.0 & 17.0 & 25.0 & 7.0 & 5.0 & 10.0 & 3.0 & 2.0 & 4.0 & $<0.001$ \\
\hline \multirow{5}{*}{ Vitals } & Systolic & 120.0 & \begin{tabular}{|l|}
115.0 \\
\end{tabular} & 130.0 & 120.0 & 110.0 & 135.0 & 120.0 & 110.0 & 145.0 & 90.0 & 85.0 & 90.0 & $<0.001$ \\
\hline & Diastolic & 80.0 & 70.0 & 80.0 & 80.0 & 70.0 & 90.0 & 80.0 & 70.0 & 90.0 & 55.0 & 50.0 & 60.0 & $<0.001$ \\
\hline & $\mathrm{RR}$ & 16.0 & 14.0 & 18.0 & 16.0 & 12.0 & 18.0 & 30.0 & 22.0 & 30.0 & 32.0 & 30.0 & 34.0 & $<0.001$ \\
\hline & Pulse & 92.0 & 84.0 & 96.0 & 98.0 & 94.0 & 100.0 & 100.0 & 98.0 & 102.0 & 106.0 & 102.0 & 110.0 & $<0.001$ \\
\hline & Temperature & 99.0 & 98.6 & 100.0 & 100.8 & 100.3 & 101.3 & 101.0 & 100.3 & 101.4 & 101.0 & 100.0 & 102.0 & $<0.001$ \\
\hline \multirow{7}{*}{ CBC } & HB & 11.8 & 10.6 & 12.6 & 11.2 & 10.5 & 12.0 & 10.9 & 10.4 & 11.8 & 11.9 & 10.6 & 13.8 & 0.002 \\
\hline & WBC & 6.4 & .0 & 9.5 & 7.8 & 5.8 & 10.4 & 11.0 & 9.9 & 13.2 & 14.7 & 13.9 & 16.7 & $<0.001$ \\
\hline & PLT & 297.0 & 212.5 & 368.0 & 302.0 & 273.0 & 378.0 & 309.0 & 289.0 & 401.0 & 378.0 & 276.0 & 490.0 & 0.001 \\
\hline & PMNS & 63.6 & 54.4 & 78.2 & 46.9 & 43.9 & 56.9 & 56.3 & 45.7 & 65.2 & 70.9 & 65.0 & 75.0 & $<0.001$ \\
\hline & LYMPHO & 29.5 & 23.4 & 34.6 & 18.5 & 16.3 & 21.0 & 19.4 & 15.8 & 21.0 & 16.1 & 12.3 & 16.5 & $<0.001$ \\
\hline & BASOPHILS & 2.0 & 0.0 & 2.0 & 3.0 & 3.0 & 4.0 & 3.0 & 3.0 & 5.0 & 3.0 & 3.0 & 6.0 & $<0.001$ \\
\hline & EOSINOPHILS & 1.0 & 0.0 & 2.0 & 3.0 & 2.0 & 4.0 & 3.0 & 2.0 & 4.0 & 3.0 & 2.0 & 4.0 & $<0.001$ \\
\hline \multirow{16}{*}{ Bio-Chemistry } & Bilirubin & 0.6 & 0.4 & 0.8 & 0.4 & 0.4 & 0.5 & 0.4 & 0.3 & 0.5 & 0.4 & 0.3 & 0.6 & $<0.001$ \\
\hline & ALT & 22.0 & 16.0 & 26.0 & 31.0 & 23.0 & 37.0 & 32.0 & 24.0 & 36.0 & 29.0 & 24.0 & 54.0 & $<0.001$ \\
\hline & AST & 27.5 & 20.0 & 34.0 & 33.0 & 24.0 & 43.0 & 32.0 & 24.0 & 43.0 & 36.0 & 27.0 & 56.0 & $<0.001$ \\
\hline & ALK PHOSPHATE & 92.0 & 73.5 & 118.0 & 87.0 & 68.0 & 99.0 & 89.0 & 67.0 & 99.0 & 87.0 & 66.0 & 100.0 & 0.025 \\
\hline & Albumin & 4.4 & 3.9 & 4.7 & 3.7 & 3.2 & 4.0 & 3.7 & 3.6 & 3.9 & 3.6 & 3.2 & 3.9 & $<0.001$ \\
\hline & Urea & 33.0 & 25.0 & 39.0 & 56.0 & 45.0 & 75.0 & 54.0 & 43.0 & 89.0 & 48.0 & 34.0 & 78.0 & $<0.001$ \\
\hline & BUN & 15.0 & 12.0 & 17.0 & 21.0 & 17.0 & 30.0 & 21.0 & 19.0 & 48.0 & 20.0 & 17.0 & 35.0 & $<0.001$ \\
\hline & Creatinine & 1.2 & 0.9 & 1.3 & 0.7 & 0.5 & 0.9 & 0.6 & 0.5 & 1.3 & 0.8 & 0.4 & 1.1 & $<0.001$ \\
\hline & Urine PH & 6.0 & 5.8 & 6.1 & 5.7 & 5.5 & 6.0 & 5.6 & 5.5 & 6.0 & 5.7 & 5.5 & 6.0 & $<0.001$ \\
\hline & \begin{tabular}{|l} 
Blood sugar level \\
\end{tabular} & 109.0 & 99.5 & 154.0 & 102.0 & 98.0 & 109.0 & 109.0 & 100.0 & 129.0 & 104.0 & 99.0 & 114.0 & 0.020 \\
\hline & D-Dimer & 296.5 & 264.0 & 349.0 & 678.0 & 550.0 & 879.0 & 1876.0 & 1056.0 & 2817.0 & 4590.0 & 3425.0 & 6234.0 & $<0.001$ \\
\hline & LDH & 213.0 & 178.0 & 256.0 & 238.0 & 209.0 & 301.0 & 278.0 & 209.0 & 301.0 & 390.0 & 309.0 & 473.0 & $<0.001$ \\
\hline & CPK & 128.0 & \begin{tabular}{|l|}
103.0 \\
\end{tabular} & 180.0 & 134.0 & 109.0 & 156.0 & 145.0 & 124.0 & 176.0 & 189.0 & 132.0 & 231.0 & $<0.001$ \\
\hline & CKMB & 16.0 & 12.0 & 20.0 & 23.0 & 20.0 & 28.0 & 24.0 & 20.0 & 28.0 & 34.0 & 27.0 & 59.0 & $<0.001$ \\
\hline & CRP & 0.3 & 0.2 & 0.4 & 0.8 & 0.5 & 1.5 & 7.2 & 2.5 & 13.2 & 19.6 & 17.2 & 21.3 & $<0.001$ \\
\hline & Serum Ferritin & 173.5 & 97.5 & 226.5 & 789.0 & 567.0 & 1029.0 & 2301.0 & 987.0 & 3009.0 & 3982.0 & 3425.0 & 4029.0 & $<0.001$ \\
\hline \multirow{6}{*}{ Serum Electrolytes } & $\mathrm{Na}+$ & 143.0 & 139.0 & 145.5 & 134.0 & 130.0 & 138.0 & 135.0 & 134.0 & 138.0 & 134.0 & 132.0 & 139.0 & $<0.001$ \\
\hline & $\mathrm{K}+$ & 4.2 & 3.8 & 4.4 & 4.4 & 3.9 & 4.7 & 4.5 & 3.9 & 4.9 & 4.2 & 3.8 & 4.9 & 0.001 \\
\hline & CL- & 102.0 & 96.0 & 105.0 & 102.0 & 100.0 & 106.0 & 104.0 & 100.0 & 107.0 & 102.0 & 99.0 & 103.0 & 0.001 \\
\hline & MG+ & 1.9 & 1.7 & 2.3 & 2.2 & 1.8 & 2.3 & 2.1 & 2.0 & 2.5 & 2.3 & 1.9 & 2.5 & $<0.001$ \\
\hline & $\mathrm{Ca}+$ & 9.3 & 8.8 & 9.5 & 9.2 & 8.6 & 9.7 & 8.8 & 8.3 & 9.2 & 9.3 & 8.6 & 10.0 & 0.016 \\
\hline & $\mathrm{PO} 4+$ & 3.6 & 3.0 & 4.1 & 3.8 & 3.2 & 4.2 & 4.2 & 3.2 & 4.8 & 3.9 & 2.9 & 4.9 & 0.006 \\
\hline \multirow{3}{*}{ Bleeding Order } & PT & 12.0 & 11.0 & 13.0 & 13.0 & 12.0 & 14.0 & 13.0 & 12.0 & 14.0 & 12.0 & 12.0 & 14.0 & $<0.001$ \\
\hline & APTT & 35.0 & 33.0 & 36.0 & 32.0 & 30.0 & 33.0 & 32.0 & 30.0 & 33.0 & 31.0 & 30.0 & 34.0 & $<0.001$ \\
\hline & INR & 1.1 & 1.0 & 1.1 & 1.1 & 1.1 & 1.2 & 1.1 & 1.0 & 1.2 & 1.1 & 1.0 & 1.2 & $<0.001$ \\
\hline
\end{tabular}

Table III: D-dimer and serum ferritin levels as discriminatory marker for severity of disease.

\begin{tabular}{|c|c|c|c|c|c|c|c|c|c|c|c|}
\hline & \multirow{3}{*}{ Lab values } & \multicolumn{10}{|l|}{ Group } \\
\hline & & \multicolumn{2}{|c|}{$\begin{array}{c}\text { Mild } \\
(n=324)\end{array}$} & \multicolumn{2}{|c|}{$\begin{array}{c}\text { Moderate } \\
(n=73)\end{array}$} & \multicolumn{2}{|c|}{$\begin{array}{l}\text { Severe } \\
(n=25)\end{array}$} & \multicolumn{2}{|c|}{$\begin{array}{l}\text { Critical } \\
(n=23)\end{array}$} & \multicolumn{2}{|c|}{$\begin{array}{c}\text { Total } \\
(n=445)\end{array}$} \\
\hline & & $\mathrm{n}$ & $\%$ & $n$ & $\%$ & $\mathbf{n}$ & $\%$ & $\mathbf{N}$ & $\%$ & $\mathrm{n}$ & $\%$ \\
\hline \multirow{4}{*}{$\begin{array}{l}\text { D. Dimer } \\
\text { (ng/mL) }\end{array}$} & $\leq 450$ & 320 & 98.8 & 2 & 2.7 & 0 & 0.0 & 0 & 0.0 & 322 & 72.4 \\
\hline & $451-990$ & 4 & 1.2 & 55 & 75.3 & 4 & 16.0 & 0 & 0.0 & 63 & 14.2 \\
\hline & $991-2900$ & 0 & 0.0 & 16 & 21.9 & 15 & 60.0 & 3 & 13.0 & 34 & 7.6 \\
\hline & $>2900$ & 0 & 0.0 & 0 & 0.0 & 6 & 24.0 & 20 & 87.0 & 26 & 5.8 \\
\hline \multirow{4}{*}{$\begin{array}{l}\text { S. Ferritin } \\
(\mu \mathrm{g} / \mathrm{mL})\end{array}$} & $\leq 300$ & 301 & 92.9 & 2 & 2.7 & 0 & 0.0 & 0 & 0.0 & 303 & 68.1 \\
\hline & $301-1000$ & 23 & 7.1 & 49 & 67.1 & 7 & 28.0 & 0 & 0.0 & 79 & 17.8 \\
\hline & $1001-3100$ & 0 & 0.0 & 21 & 28.8 & 14 & 56.0 & 3 & 13.0 & 38 & 8.5 \\
\hline & $>3100$ & 0 & 0.0 & 1 & 1.4 & 4 & 16.0 & 20 & 87.0 & 25 & 5.6 \\
\hline
\end{tabular}

As suggested by Zhang et al, drug toxicity, hypoxia or cytokine storm may contribute to transient liver injuries in COVID-19 patients. ${ }^{20}$ The over-expression of the ACE-2 receptors in the tubular cells of the kidney alongwith a raised BUN and creatinine also point to the vulnerability of CKD patients to the SARS-CoV-2 virus. ${ }^{21}$
Among the inflammatory markers, D-dimers, serum ferritin and CRP were significantly raised in the moderate, severe and critical cases with higher levels suggesting a more serious disease. ${ }^{22}$ In a local study, CRP, ferritin and LDH were raised in about $90 \%$ of the severe cases and in about $70 \%$ of the mild category patients, as well. ${ }^{15}$ This was in slight opposition to the present study as these markers were found to 
be normal in the mild category patients. Cardiac biomarkers like LDH, CPK and CK-MB were also linked with the severity of the disease; the more serious the disease, the raised the marker levels. This enzyme assay helped in recognizing myocardial injury in COVID-19 patients and we inferred that if it is done at an earlier stage of the disease, it could prevent cardiac failure in these patients. ${ }^{23}$

Duration of hospital stay had a very different pattern. Patients falling in the mild to moderate category had a longer duration of stay in the hospital compared to the patients having severe and critical disease. Patients with more serious disease succumbed to their illness earlier, mainly because of underlying comorbidities, ventilator associated lung injury, multi-organ failure and an exaggerated immune response.

This study had certain limitations. The data collected from the patients was during the first wave of COVID-19, so clinical and laboratory data that was found significant then may have become redundant by the time of its publication. Secondly, certain markers like IL-6, pro-calcitonin were not readily available at that time so their relationship to the severity of disease could not be assessed. Thirdly, it was conducted on a small number of patients belonging to a specified area, so the findings cannot be generalised to the whole Pakistani population.

\section{CONCLUSION}

This single centered study conducted during the first wave of the pandemic showed a significant interplay of clinical, laboratory and radiological factors responsible for disease severity. Advancing age, smoking and presence of comorbid conditions, mainly hypertension and diabetes, were found to have a significant association with the severity of COVID-19 infection. D-dimers, serum ferritin and cardiac enzymes were helpful markers in assessing the severity of disease.

\section{ETHICAL APPROVAL:}

Ethical approval was taken from the Institutional Review Board (IRB) of King Edward Medical University, Lahore prior to initiation of the study.

\section{PATIENTS' CONSENT:}

Informed written consents to be enrolled for the study purpose and data collection were taken from all the patients having mild and moderate disease and from the attendants in cases of severe and critical disease

\section{CONFLICT OF INTEREST:}

The authors declared no conflict of interest.

\section{AUTHORS' CONTRIBUTION:}

TN: Conceived designed the study, paper write-up.

BA: Data collection and statistical analysis.

BS: Proof-reading and discussion.

FS, KN, MN: Result compilation.

\section{REFERENCES}

1. COVID-19 coronavirus pandemic worldometer. Last updated April 12, 2021, 07:43 GMT.

2. Hassan S, Sheikh FN, Jamal S, Ezeh JK, Akhtar A. Coronavirus (COVID-19): A review of clinical features, diagnosis, and treatment. Cureus 12(3):e7355. doi:10.7759/cureus. 7355.

3. Wu Z, McGoogan JM. Characteristics of and important lessons from the coronavirus disease 2019 (COVID-19) outbreak in China: Summary of a report of 72,314 cases from the Chinese centre for disease control and prevention. JAMA 2020; 323(13):1239-42. doi: 10.1001/jama.2020. 2648.

4. Epidemiological characteristics of the new coronavirus pneumonia. Epidemiology group of the new coronavirus pneumonia emergency response mechanism of the chinese center for disease control and prevention. Chin J Epidemiol 2020; 41:145-151.

5. Shi H, Han X, Jiang N, Cao Y, Alwalid O, Gu J, et al. Radiological findings from 81 patients with COVID-19 pneumonia in Wuhan, China: A descriptive study. Lancet Infect Dis 2020; 20(4):425-34. doi: 10.1016/S1473-3099(20)30086-4.

6. Adhikari SP, Meng S, Wu Y, Mao Y, Ye R, Wang Q, et al. Epidemiology, causes, clinical manifestations and diagnosis, prevention and control of coronavirus disease (COVID-19) during the early outbreak period: A scoping review. Infect Dis Poverty 2020; 9(1):29. doi: 10.1186/s40249-02000646-x.

7. Li Q, Guan X, Wu P, Wang X, Zhou L, Tong Y, et al. Early transmission dynamics in Wuhan, China, of novel coronavirus-infected pneumonia. N Engl J Med 2020; 382(13): 1199-1207. doi: 10.1056/NEJMoa2001316.

8. Liu K, Fang YY, Deng Y, Liu W, Wang M, Ma J, et al. Clinical characteristics of novel coronavirus cases in tertiary hospitals in Hubei province. Chin Med J (Engl) 2020; 133(9): 1025-31. doi: 10.1097/CM9.0000000000000744.

9. Shi H, Han X, Jiang N, Cao Y, Alwalid O, Gu J, et al. Radiological findings from 81 patients with COVID-19 pneumonia in Wuhan, China: A descriptive study. Lancet Infect Dis 2020; 20(4):425-34. doi: 10.1016/S1473-3099(20)30086-4.

10. Wang D, Hu B, Hu C, Zhu F, Liu X, Zhang J, et al. Clinical characteristics of 138 hospitalised patients with 2019 novel coronavirus-infected pneumonia in Wuhan, China. JAMA 2020; 323(11):1061-9. doi: 10.1016/S1473-3099(20) 30086-4.

11. Fang L, Karakiulakis G, Roth M. Are patients with hypertension and diabetes mellitus at increased risk for COVID-19 infection? Lancet Respir Med 2020; 8(4):21. doi: 10.1016/S2213-2600(20)30116-8.

12. Iqtadar S, Ahmad J, Akhtar MS, Ghani U. COVID-19 Standard Operating Procedures and Guidelines. Government of Pakistan, Ministry of National Health Services, Regulations and Coordination. 2nd Revision, 28th March, 2020, pg 15-17

13. Liu X, Yue X, Liu F, Wei L, Chu Y, Bao H, et al. Analysis of clinical features and early warning signs in patients with severe COVID-19: A retrospective cohort study. PLOS ONE 2020; 15(6):e0235459. doi.org/10.1371/journal.pone.0235 459. 
14. Paudel SS. A meta-analysis of 2019 novel coronavirus patient clinical characteristics and comorbidities. Research Square. 2020. 10.21203/rs.3.rs-21831/v1 [accessed on April 18,2020 , http://www.researchsquare.com/article/rs- 21831/v1]

15. Asghar MS, Kazmi SJH, Khan NA, Akram M, Khan SA, Rasheed $U$, et al. Clinical profiles, characteristics and outcomes of the first 100 admitted COVID-19 patients in Pakistan: A single-centre retrospective study in a tertiary care hospital of Karachi. Cereus 2020; 12(6):e8712.doi: 10.7759?cereus.8712.

16. BCCDC. COVID-19 vulnerable populations. British Columbia Centre for Disease Control. 2020. [Accessed on April 18, 2020 ,

http://www.bccdc.ca/health-info/diseases-conditions/covid-1 9/vulnerable-populations]

17. Zhou F, Yu T, Du R, Guohui Fan MS, Liu Y, Liu Z, et al. Clinical course and risk factors for mortality of adult inpatients with COVID-19 in Wuhan, China: A retrospective cohort study. Lancet 2020; 395(10229):pg1054-62. doi.org/10. 1016/S0140-6736 (20)30566-3.

18. Wang B, Li R, Lu Z, Huang Y. Does comorbidity increase the risk of patients with COVID-19: Evidence from meta-analysis. Aging (Albany NY) 2020; 12(7):6049-6057. doi10.18632/aging.103000.

19. Zhao Y, Nie H, Hu K, Wu X, Zhang Y, Wang M, et al. Abnormal immunity of non-survivors with COVID-19: Predictors for mortality. Infect Dis Poverty 2020; 9(1):108. doi.org/10.1186/s40249-020-00723-1.

20. Zhang C, Shi L, Wang FS. Liver injury in COVID-19: Management and challenges. Lancet Gastroenterol Hepatol 2020; 5(5):428-30. doi: 10.1016/S2468-1253(20)30057-1.

21. Fan C, Li K, Ding Y, Lu WL, Wang J. ACE2 expression in kidney and testis may cause kidney and testis damage after 2019-nCoV infection. MedRxiv 2020; doi: 10.1101/2020. 02.12.20022418.

22. Yao Y, Cao J, Wang Q, Shi Q, Liu K, Luo Z, et al. D-dimer as a biomarker for disease severity and mortality in COVID-19 patients: A case control study. J Intensive Care 2020; 8:49 doi.org/10.1186/s40560-020-00466-z.

23. Li X. Application value of myocardial enzyme spectrum detection in emergency diagnosis [j]. Smart Health 2019; 5(32):96-7. 